\title{
Prospects for the future of epidermal growth factor receptor-tyrosine kinase inhibitors in combination with bevacizumab
}

\author{
Yasuhiro Chikaishi ${ }^{1,2}$, Fumihiro Tanaka ${ }^{2}$, Yohei Honda ${ }^{1}$, Masaaki Inoue ${ }^{1}$, Junichi Yoshida ${ }^{1}$, Masao Tanaka ${ }^{1}$ \\ ${ }^{1}$ Department of Chest Surgery, Shimonoseki City Hospital, Kouyouchou, Shimonoseki, Japan; ${ }^{2}$ Second Department of Surgery, School of Medicine, \\ University of Occupational and Environmental Health, Yahatanishi-ku, Kitakyushu, Japan \\ Correspondence to: Yasuhiro Chikaishi. Department of Chest Surgery, Shimonoseki City Hospital, 1-13-1 Kouyouchou, Shimonoseki 750-8520, Japan. \\ Email: cywmd0k2@med.uoeh-u.ac.jp. \\ Comment on: Saito H, Fukuhara T, Furuya N, et al. Erlotinib plus bevacizumab vs. erlotinib alone in patients with EGFR-positive advanced non- \\ squamous non-small-cell lung cancer (NEJ026): interim analysis of an open-label, randomised, multicentre, phase 3 trial. Lancet Oncol 2019;20:625-35.
}

Submitted Sep 17, 2019. Accepted for publication Jan 06, 2020.

doi: $10.21037 /$ tcr.2020.01.24

View this article at: http://dx.doi.org/10.21037/tcr.2020.01.24

Lung cancer is a major cause of cancer-related death. The main initial treatment for advanced and recurrent lung cancer is systemic chemotherapy. The survival rates for first line platinum-based chemotherapy, which is the cornerstone chemotherapy for non-small cell lung cancer (NSCLC), are poor (1). However, the survival rate of NSCLC, particularly adenocarcinoma, has recently improved with the development of strategies involving moleculartargeted therapies, angiogenesis inhibitors, and checkpoint inhibitors. The treatment strategy for NSCLC is evolving.

Since the approval of gefitinib, which was the first epidermal growth factor receptor (EGFR) inhibitor to reach the market, EGFR-tyrosine kinase inhibitors (TKIs) have displayed efficacy in the treatment in patients with mutated EGFR $(2,3)$. The progression-free survival (PFS) of first- and second-generation EGFR-TKIs (e.g., gefitinib, erlotinib, afatinib) is approximately 1 year, and acquired resistance (AR) is mostly inevitable (4). Meanwhile, the third-generation EGFR-TKI osimertinib prolonged PFS to 18.9 months (5). Treatment with EGFR-TKIs has progressed rapidly in this decade. Bevacizumab, a monoclonal antibody against vascular endothelial growth factor (VEGF), had prolonged PFS for NSCLC without squamous cell carcinoma in combination with platinum doublets (6). Recently, some clinical studies of EGFR-TKI treatment combined with bevacizumab were performed to improve prognosis for EGFR-positive NSCLC (7-10).

In patients with EGFR-mutated non-squamous NSCLC, erlotinib plus bevacizumab improved PFS compared with the effects of erlotinib alone in a phase III trial (NEJ026) conducted by Saito et al. (7). In the NEJ026 study, 228 patients with non-squamous NSCLC harboring common EGFR mutations were randomized to receive either oral erlotinib $150 \mathrm{mg}$ per day plus intravenous bevacizumab $15 \mathrm{mg} / \mathrm{kg}$ once every 21 days or erlotinib $150 \mathrm{mg}$ per day alone. In the interim analysis, the median PFS of patients in the erlotinib plus bevacizumab group was 16.9 months, compared with 13.3 months for patients in the erlotinib group [hazard ratio (HR) $0.606,95 \%$ confidence interval (CI): $0.417-0.877 ; \mathrm{P}=0.016$ ). The objective response rates in the combination and monotherapy arms were $72 \%$ and $66 \%(\mathrm{P}=0.31)$, respectively, and the disease control rates were $95 \%$ and $96 \%(\mathrm{P}=0.52)$, respectively. All patients in the erlotinib plus bevacizumab group had a decrease in tumor size $v s$. baseline, although a few patients in the erlotinib group experienced such an increase. Subgroup analysis by EGFR genomic aberration type revealed that the median PFS was better in patients with the L858R mutation in the erlotinib plus bevacizumab group than in the erlotinib group (17.4 vs. 13.7 months, HR 0.57, 95\% CI: 0.33-0.97). Conversely, no differences in survival were found between the erlotinib plus bevacizumab and erlotinib alone groups in patients with exon 19 deletion (16.6 vs. 12.4 months, HR $0.69,95 \%$ CI: 0.41-1.16). In subgroup analyses by CNS metastasis and pleural effusion (PE), the HR for PFS was better the erlotinib plus bevacizumab 
group than the erlotinib group, although these differences were not statistically significant (HR $0.78,95 \%$ CI: $0.42-1.43$ and HR $0.58,95 \%$ CI: $0.34-1.02$, respectively). Adverse events of grade 3 or worse were more common in the erlotinib plus bevacizumab group than in the erlotinib group ( $88 \%$ and $46 \%$, respectively). The median duration of erlotinib administration was 405 days (range, 5-807 days) in the erlotinib plus bevacizumab group, vs. 364 days (range, 43-736 days) in the erlotinib group. The most commonly reported adverse events causing bevacizumab discontinuation were proteinuria [11 (33\%) patients], hemorrhage [excluding pulmonary hemorrhage; 3 (9\%) patients], and hepatic dysfunction [3 (9\%) patients]. The most commonly reported adverse events resulting in erlotinib discontinuation were rash $[8(7 \%)$ patients in the erlotinib plus bevacizumab group and $8(7 \%)$ patients in the erlotinib group].

To the best of our knowledge, NEJ026 is the first multicenter phase 3 study to compare the efficacy of bevacizumab plus an EGFR-TKI with an EGFR-TKI alone in patients with NSCLC. Regarding the EGFR genomic aberration type, some studies (11-13) reported better efficacy in patients with exon 19 deletion than in those carrying the L858R mutation. A greater number of samples may result in different results, but erlotinib plus bevacizumab may have better efficacy for patients with the L858R mutation than in those with exon 19 deletion. Regarding phase 2 trials, some studies assessed bevacizumab and EGFR-TKI combination treatment $(5,8-10,14)$. Kitagawa et al. reported a phase 2 study (9) comparing gefitinib plus bevacizumab to single agent of gefitinib in patients with EGFR-positive NSCLC. This study concluded that PFS was worse in the gefitinib with bevacizumab group than in the gefitinib group (5.4 and 15.1 months, respectively); and hence, there was not able to proceed to a phase III trial. However, comparisons with other results could be difficult because this study was small $(\mathrm{n}=16)$. Hata $e t$ al. reported a phase 2 study (10) assessing afatinib plus bevacizumab after AR to EGFR-TKIs in patients with EGFR-mutant NSCLC. This study reported that afatinib plus bevacizumab was clinically effective and safe after AR to EGFR-TKIs, showing that it could be a therapeutic strategy option for patients carrying the T790M mutation. However, this was a single-arm study opposed to a controlled trial.

Osimertinib, a third-generation EGFR-TKI, was linked to better prognosis than platinum doublet chemotherapy in patients with EGFR T790M-mutated lung cancer in the phase III AURA3 trial (15). Moreover, osimertinib was determined to be the best TKI in terms of PFS based on the results of the FLAURA study (5). Currently, two phase I/II studies of osimertinib plus bevacizumab are ongoing (WJOG8715L, SPIRAL II) $(8,16)$. WJOG8715 is a phase I/II study of osimertinib plus bevacizumab in patients with EGFR-mutated, T790M-positive cancer who acquired resistance on EGFR-TKIs, and SPIRAL II is a phase II trial of osimertinib plus bevacizumab in patients with nontherapy EGFR-mutated NSCLC and malignant PE and/ or pericardial effusion. Both are single-arm studies. In the future, a controlled trial of osimertinib with bevacizumab $v s$. osimertinib alone in patients with EGFR-mutated cancer could lead to improved outcomes.

Regarding CNS metastasis and PE, the NEJ026 study (7) did not reveal a significant benefit of erlotinib plus bevacizumab. However, erlotinib could be more effective for the treatment for CNS metastases than gefitinib or afatinib $(17,18)$. In vivo data indicated that anti-VEGF treatments could be useful for controlling malignant PE (19). Moreover, we previously reported the effect of erlotinib with bevacizumab to brain metastases in patients with NSCLC (20). In NEJ026, the efficacy of erlotinib plus bevacizumab against CNS metastasis and PE tended to be better than that of erlotinib alone (HR 0.78, 95\% CI: $0.42-1.43$ and HR $0.58,95 \%$ CI: 0.34-1.02, respectively). Osimertinib has better efficacy against CNS metastases in patients with untreated EGFR-mutated NSCLC than gefitinib or erlotinib (21). Osimertinib plus bevacizumab is expected to have greater efficacy against CNS metastases.

Recently, immune checkpoint inhibitors (ICIs) have emerged as promising alternative treatments for NSCLC (22). However, a recent retrospective study found that patients with oncogenic driver mutations, such as EGFR and anaplastic lymphoma kinase (ALK), tend to display reduced responses to ICIs regarding objective response rates and PFS compared with the effects in patients with wild-type EGFR and ALK-negative cancer (23). The reason for this finding is unclear, and the therapeutic strategy of EGFRTKI with ICIs has not been reported. Conversely, in the IMpower150 study, ICIs in combination with bevacizumab were effective against NSCLC, including lesions with EGFR or ALK genetic alterations (24). Regarding the use of ICIs for treating NSCLC featuring oncogenic driver mutations such as EGFR and ALK, further research is needed to validate the clinical biomarkers involved in the response to ICI therapy.

At present, several treatment strategies are available for 
EGFR-mutant NSCLC, such as osimertinib alone, firstor second-generation EGFR-TKIs alone, EGFR-TKIs combined with bevacizumab, and EGFR-TKIs combined with platinum doublets. We must optimize these treatment strategies for patients with EGFR-mutant NSCLC. In the future, we hope to develop more efficient new-generation EGFR-TKIs or treatment strategies for patients with EGFR-mutant NSCLC.

\section{Acknowledgments}

Funding: None.

\section{Footnote}

Provenance and Peer Review: This article was commissioned and reviewed by the Section Editor Wei Xu (Division of Respiratory Disease, Department of Geriatrics, the First Affiliated Hospital of Nanjing Medical University, Nanjing, China).

Conflicts of Interest: All authors have completed the ICMJE uniform disclosure form (available at http://dx.doi. org/10.21037/tcr.2020.01.24). The authors have no conflicts of interest to declare.

Ethical Statement: The authors are accountable for all aspects of the work in ensuring that questions related to the accuracy or integrity of any part of the work are appropriately investigated and resolved.

Open Access Statement: This is an Open Access article distributed in accordance with the Creative Commons Attribution-NonCommercial-NoDerivs 4.0 International License (CC BY-NC-ND 4.0), which permits the noncommercial replication and distribution of the article with the strict proviso that no changes or edits are made and the original work is properly cited (including links to both the formal publication through the relevant DOI and the license). See: https://creativecommons.org/licenses/by-nc-nd/4.0/.

\section{References}

1. Grossi F, Kubota K, Cappuzzo F, et al. Future scenarios for the treatment of advanced non-small cell lung cancer: focus on taxane-containing regimens. Oncologist 2010;15:1102-12.

2. Maemondo M, Inoue A, Kobayashi K, et al. Gefitinib or chemotherapy for non-small-cell lung cancer with mutated EGFR. N Engl J Med 2010;362:2380-8.

3. Mitsudomi T, Kosaka T, Endoh H, et al. Mutations of the epidermal growth factor receptor gene predict prolonged survival after gefitinib treatment in patients with nonsmall-cell lung cancer with postoperative recurrence. J Clin Oncol 2005;23:2513-20.

4. Lee CK, Wu YL, Ding PN, et al. Impact of Specific Epidermal Growth Factor Receptor (EGFR) Mutations and Clinical Characteristics on Outcomes After Treatment With EGFR Tyrosine Kinase Inhibitors Vs. Chemotherapy in EGFR-Mutant Lung Cancer: a Meta-Analysis. J Clin Oncol 2015;33:1958-65.

5. Soria JC, Ohe Y, Vansteenkiste J, et al. FLAURA Investigators. Osimertinib in Untreated EGFR-Mutated Advanced Non-Small-Cell Lung Cancer. N Engl J Med 2018;378:113-25.

6. Sandler A, Gray R, Perry MC, et al. Paclitaxel-carboplatin alone or with bevacizumab for non-small-cell lung cancer. N Engl J Med 2006;355:2542-50.

7. Saito H, Fukuhara T, Maemondo M, et al. Erlotinib plus bevacizumab vs. erlotinib alone in patients with EGFRpositive advanced non-squamous non-small-cell lung cancer (NEJ026): interim analysis of an open-label, randomised, multicentre, phase 3 trial. Lancet Oncol 2019;20:625-35.

8. Akamatsu H, Teraoka S, Morita S, et al. Phase I/II Study of Osimertinib With Bevacizumab in EGFR-mutated, T790M-positive Patients With Progressed EGFR-TKIs: West Japan Oncology Group 8715L (WJOG8715L). Clin Lung Cancer 2019;20:e492-4.

9. Kitagawa C, Mori M, Ichinose Y, et al. Gefitinib Plus Bevacizumab vs. Gefitinib Alone for EGFR Mutant Non-squamous Non-small Cell Lung Cancer. In Vivo 2019;33:477-82.

10. Hata A, Katakami N, Kaji R, et al. Afatinib plus bevacizumab combination after acquired resistance to EGFR tyrosine kinase inhibitors in EGFR-mutant nonsmall cell lung cancer: Multicenter, single-arm, phase 2 trial (ABC Study). Cancer 2018;124:3830-8.

11. Yang JC, $W u$ YL, Schuler M, et al. Afatinib vs. cisplatinbased chemotherapy for EGFR mutation-positive lung adenocarcinoma (LUX-Lung 3 and LUX-Lung 6): analysis of overall survival data from two randomised, phase 3 trials. Lancet Oncol 2015;16:141-51.

12. Rosell R, Carcereny E, Gervais R, et al. Erlotinib vs. standard chemotherapy as first-line treatment for European patients with advanced EGFR mutation-positive 
non-small-cell lung cancer (EURTAC): a multicentre, open-label, randomised phase 3 trial. Lancet Oncol 2012;13:239-46.

13. Sheng M, Wang F, Zhao Y, et al. Comparison of clinical outcomes of patients with non-small-cell lung cancer harbouring epidermal growth factor receptor exon 19 or exon 21 mutations after tyrosine kinase inhibitors treatment: a meta-analysis. Eur J Clin Pharmacol 2016;72:1-11.

14. Seto T, Kato T, Nishio M, et al. Erlotinib alone or with bevacizumab as first-line therapy in patients with advanced non-squamous non-small-cell lung cancer harbouring EGFR mutations (JO25567): an open-label, randomised, multicentre, phase 2 study. Lancet Oncol 2014;15:1236-44.

15. Mok TS, Wu Y-L, Ahn M-J, et al, Osimertinib or Platinum-Pemetrexed in EGFR T790M-Positive Lung Cancer. N Engl J Med 2017;376:629-40.

16. Hiranuma O, Uchino J, Yamada T, et al. Rationale and Design of a Phase II Trial of Osimertinib Combined With Bevacizumab in Patients With Untreated Epidermal Growth Factor Receptor-mutated Non-small-cell Lung Cancer and Malignant Pleural and/or Pericardial Effusion (SPIRAL II Study). Clin Lung Cancer 2019;20:e402-6.

17. Togashi Y, Masago K, Masuda S, et al. Cerebrospinal fluid concentration of gefitinib and erlotinib in patients with non-small cell lung cancer. Cancer Chemother Pharmacol 2012;70:399-405.

18. Cross DA, Ashton SE, Ghiorghiu S, et al. AZD9291, an irreversible EGFR TKI, overcomes T790M-mediated resistance to EGFR inhibitors in lung cancer. Cancer

Cite this article as: Chikaishi Y, Tanaka F, Honda Y, Inoue M, Yoshida J, Tanaka M. Prospects for the future of epidermal growth factor receptor-tyrosine kinase inhibitors in combination with bevacizumab. Transl Cancer Res 2020;9(3):1307-1310. doi: 10.21037/tcr.2020.01.24
Discov 2014;4:1046-61.

19. Yano S, Herbst RS, Shinohara H, et al. Treatment for malignant pleural effusion of human lung adenocarcinoma by inhibition of vascular endothelial growth factor receptor tyrosine kinase phosphorylation. Clin Cancer Res 2000;6:957-65.

20. Chikaishi Y, Kanayama M, Tanaka F, et al. Effect of erlotinib plus bevacizumab on brain metastases in patients with non-small cell lung cancer. Ann Transl Med 2018;6:401.

21. Reungwetwattana T, Nakagawa K, Cho BC, et al. CNS Response to Osimertinib Vs. Standard Epidermal Growth Factor Receptor Tyrosine Kinase Inhibitors in Patients With Untreated EGFR-Mutated Advanced Non-SmallCell Lung Cancer. J Clin Oncol 2018. [Epub ahead of print].

22. Yoneda K, Ichiki Y, Tanaka F, et al. Immune Checkpoint Inhibitors (ICIs) in Non-Small Cell Lung Cancer (NSCLC). J UOEH 2018;40:173-89.

23. Gainor JF, Shaw AT, Sequist LV, et al. EGFR Mutations and ALK Rearrangements Are Associated with Low Response Rates to PD-1 Pathway Blockade in Non-Small Cell Lung Cancer: a Retrospective Analysis. Clin Cancer Res 2016;22:4585-93.

24. Reck M, Mok TSK, Nishio M, et al. Atezolizumab plus bevacizumab and chemotherapy in non-small-cell lung cancer (IMpower150):key subgroup analyses of patients with EGFR mutations or baseline liver metastases in a randomised, open-label phase 3 trial. Lancet Respir Med 2019;7:387-401. 